\title{
Editorial / Editorial / Editorial
}

\section{La investigación en salud en tiempos de pandemia}

Wilson Bautista-Molano, MD, Ph. D.*

DOI: https://doi.org/10.18359/rmed.5786

La situación epidemiológica actual en relación con la pandemia ha modificado sustancialmente el desarrollo y la continuidad de los proyectos de investigación en la gran mayoría de sectores del conocimiento, incluida el área de la salud. A principios de este año, el síndrome respiratorio agudo severo asociado al coronavirus 2 (sARsCOV-2 por sus siglas en inglés) fue declarado pandemia y emergencia sanitaria global por parte de la Organización Mundial de la Salud (OMs). Más de 48 millones de contagios hasta finales de octubre, con más de 1,2 millones de muertes han sido reportadas por el SARS-COV-2, llamado COVID-19 (1). Un número importante de medidas restrictivas de movilidad se han implementado por parte de autoridades locales y nacionales con el fin de contener la tasa de contagio y evitar el colapso de los sistemas de salud especialmente en Latinoamérica (2) y en países de ingresos medios y bajos (3). Con base en lo anterior, se crea la necesidad de disponer de modelos predictivos de la dinámica de transmisión y propagación del virus (4), que permitan la toma de medidas de intervención a gran escala.

En este escenario, la investigación biomédica en cuanto al covid-19 se ha convertido en una prioridad en los contextos académico y asistencial (5). Varias revistas científicas (6) y bases de datos (7) globales permiten el acceso abierto de las publicaciones relacionadas con la pandemia, y reconocen la necesidad e importancia de compartir información científica sobre el agente viral causante de esta condición y su impacto en la comunidad. Estas fuentes de información de acceso abierto posibilitan socializar resultados de interés y estimulan la apropiación social del conocimiento en esta área.

Hemos sido testigos de que esta pandemia puede causar interrupciones en el trabajo de nuestra comunidad de científicos e investigadores. Ha sido notorio que muchos autores, evaluadores y académicos han visto retrasada la continuidad y el avance de protocolos y proyectos de investigación en especial acerca de patologías no relacionadas con Covid. Desde el punto de vista de publicaciones académicas, uno de los principales retos y desafíos es garantizar que la comunicación de los resultados y avances científicos en el área de la salud continúe de la mejor manera posible y se mantenga durante esta situación excepcional. En este sentido, es probable que algunos investigadores puedan tener resultados novedosos y de gran mérito que requerirían de un trabajo adicional considerable para llegar a ser un artículo completo.

Sin embargo, este escenario de incertidumbre se puede constituir en un incentivo y en una ocasión sin precedentes para fortalecer el trabajo colaborativo, desarrollar proyectos de innovación e incluso mejorar las plataformas de telemedicina. Sea este un llamado para estimular la continuidad de la generación y publicación de nuevo conocimiento.

* Profesor de la Facultad de Medicina de la Universidad Militar Nueva Granada y la Universidad El Bosque. Hospital Universitario Fundación Santa Fe de Bogotá, Colombia. Correo electrónico: wilson.bautista@unimilitar.edu 


\section{Referencias}

(1) World Health Organization. Coronavirus disease (Covid-19) pandemic. Disponible en: https://www. who.int/emergencies/diseases/novel-coronavirus-2019

(2) Bautista-Molano W, Ferreyra Garrot L, Toro C. Exploring the impact of Covid-19 in Latin America. J Clin Rheumatol. 2020;26(6):218-219. Dor: https://doi. org/10.1097/RHU.0000000000001523

(3) Hodkinson B, Singh P, Gcelu A, Bautista-Molano W, Pons-Estel G, Alpízar-Rodríguez D. Navigating COVID-19 in the developing world. Clin Rheumatol. 2020;(39):2039-2042. DoI: https://doi.org/10.1007/ s10067-020-05159-4
(4) Díaz Pinzón JE. Proyección de la propagación del Covid-19 en Colombia. Rev. Med. 2020;28(1):11-20. DoI: https://doi.org/10.18359/rmed.4702

(5) Cucunubá ZM. Investigación científica prioritaria en Latinoamérica para orientar la prevención y el control de la covid-19. Biomédica. 2020;40(Supl. 2):9-13. Disponible en: https://revistabiomedica.org/ index.php/biomedica/article/view/5882/4694

(6) The New England Journal of Medicine. Coronavirus (Covid-19). Disponible en: https://www.nejm.org/ coronavirus

(7) NIH National Library of Medicine. NCBI SARS-COV-2 Resources. NIH National Institutes of Health. Coronavirus (covid-19). Disponible en: https://www.ncbi. nlm.nih.gov/sars-cov-2/ 